Full length article

\title{
Optimizing resin-dentin bond stability using a bioactive adhesive with concomitant antibacterial properties and anti-proteolytic activities
}

\author{
Ya-ping Gou ${ }^{\text {a }}$, Mohamed M. Meghil ${ }^{\mathrm{b}}$, Cesar R. Pucci ${ }^{\mathrm{c}}$, Lorenzo Breschi ${ }^{\mathrm{d}}$, David H. Pashley ${ }^{\mathrm{b}}$, \\ Christopher W. Cutler ${ }^{\mathrm{b}}$, Li-na Niu ${ }^{\mathrm{b}, \mathrm{e}}$, Ji-yao Li ${ }^{\mathrm{a}, *}$, Franklin R. Tay ${ }^{\mathrm{b}, \mathrm{e}, *}$ \\ a State Key Laboratory of Oral Diseases \& National Clinical Research Center for Oral Diseases E Department of Cariology and Endodontics West China Hospital of Stomatology, \\ Sichuan University, Chengdu, PR China \\ ${ }^{\mathrm{b}}$ The Dental College of Georgia, Augusta University, Augusta, GA, USA \\ ${ }^{\mathrm{c}}$ Department of Restorative Dentistry, Institute of Science and Technology, São Paulo State University UNESP São Jose dos Campos, São Paulo, Brazil \\ ${ }^{\mathrm{d}}$ Department of Biomedical and Neuromotor Sciences, DIBINEM, University of Bologna - Alma Mater Studiorum, Bologna, Italy \\ e State Key Laboratory of Military Stomatology E National Clinical Research Center for Oral Diseases E Shaanxi Key Laboratory of Oral Diseases, School of Stomatology, The \\ Fourth Military Medical University, Xi'an, Shaanxi, PR China
}

\section{A R T I C L E I N F O}

\section{Article history:}

Received 19 March 2018

Received in revised form 3 June 2018

Accepted 4 June 2018

Available online 6 June 2018

\section{Keywords:}

Antibacterial

Endogenous dentin proteases

Quaternary ammonium methacryloxy silane

Resin-dentin bonds

\begin{abstract}
A B S T R A C T
Secondary caries and hybrid layer degradation are two major challenges encountered in long-term resindentin bond stability. As a link between resin and dentin, adhesives that possess both antimicrobial and anti-proteolytic activities are in demand for eliminating bacteria-induced secondary caries and preventing hybrid layers from degradation. In the present study, a new quaternary ammonium methacryloxy silane (QAMS) prepared from sol-gel chemistry was incorporated into experimental adhesives to examine their antimicrobial effect and anti-proteolytic potential. This functional methacrylate resin monomer contains polymerizable methacryloxy functionalities as well as a positively-charged quaternary ammonium functionality with a long, lipophilic $-\mathrm{C}_{18} \mathrm{H}_{37}$ alkyl chain for puncturing the cell wall/membrane of surface-colonizing organisms. Antibacterial testing performed using agar diffusion test, live/dead bacterial staining and colony-forming unit counts all indicated that the QAMS-containing adhesives killed Streptococcus mutans and Actinomyces naeslundii in a dose-dependent manner via a predominant contact-killing mechanism. Gelatinolytic activity within the hybrid layers created by these adhesives was examined using in-situ zymography. Hybrid layers created with 0\% QAMS-containing adhesive exhibited intense green fluorescence emitted by the hydrolyzed fluorescein-conjugated gelatin, with 4 -fold increase in enzymatic activity compared with an experimental adhesive containing 5\% QAMS. Taken together, incorporation of 5\% QAMS in the experimental adhesive provides simultaneous antimicrobial and anti-proteolytic activities that are crucial for the maintenance of long-term resin-dentin bond integrity.
\end{abstract}

\section{Statement of Significance}

Durability of resin-dentin interfacial bond remains a clinically-significant challenge. Secondary caries caused by bacteria and the degradation of hybrid layers via endogenous dentin proteases are two important contributors to the poor resin-dentin bond durability. The present study developed a new 5\% QAMScontaining adhesive that provides simultaneous antimicrobial and dentin protease inhibition functions to extend the longevity of resin-dentin bonds.

Published by Elsevier Ltd on behalf of Acta Materialia Inc.

\footnotetext{
* Corresponding authors at: The Dental College of Georgia, Augusta University, Augusta, GA, USA (F.R. Tay).

E-mail addresses: jiyaoliscu@163.com (J.-y. Li), ftay@augusta.edu (F.R. Tay).
}

\section{Introduction}

Tooth-colored resin polymer tooth fillings are popular because of their excellent esthetics [1]. There are two major challenges associated with the long-term durability of these restorations. Secondary caries is the most prevalent cause for the replacement of 
tooth-colored fillings [2]. Partial retention of caries-infected dentin is not contraindicated in contemporary minimally-invasive dentistry $[3,4]$. Ingress of bacteria and their by-products through interfacial gaps between the tooth and the restoration may precipitate restorative failure $[5,6]$. The availability of oxygen and nutrients to the entrapped bacteria enable them to proliferate and cause secondary caries and pulpal damage over time $[7,8]$.

The other challenge in achieving longevity of bonds made by resins in dentin is the presence of endogenous proteases that are trapped within mineralized dentin matrix after odontogenesis. There is general consensus that resin-dentin bonds created by hydrophilic adhesive systems deteriorate over time [9,10]. Hybrid layers created in dentin by contemporary adhesives are unstable in aqueous environments $[9,11,12]$ due to hydrolysis of adhesive resins and degradation of demineralized collagen matrices. During the acid-etching phase of dentin bonding, endogenous dentin proteases such as matrix metalloproteinases (MMPs) and cysteine cathepsins that are trapped by apatite crystallites become exposed and activated by acidic etchants. Subsequent application of acidic monomers incorporated in etch-and-rinse or self-etch adhesives further promotes protease activities [13-15]. Activated, matrixbound MMPs and cathepsins can progressively degrade exposed collagen fibrils within the hybrid layers [16]. Entrapment of water within the resin-dentin interface provides an aqueous environment for proteolytic degradation of collagen and leaching of enzyme-degradable resinous components [17,18].

The aforementioned two challenges be concomitantly addressed before one can create long-lasting resin-dentin interfacial bonds. As a bridge between tooth fillings and the tooth substrate, dentin adhesives that possess antimicrobial and antiproteolytic activities are in demand. Chlorhexidine possesses broad spectrum antimicrobial activity [19] and anti-MMP properties [20], and has been incorporated into restorative materials [21]. Because chlorhexidine does not co-polymerize with methacrylate resin monomers, it eventually leaches out from the polymerized resin network [22]. Hence, adhesives incorporating chlorhexidine do not provide long-lasting antimicrobial and anti-proteolytic effects.

There has been fairly widespread study and use of quaternary ammonium salts (QAS) as antimicrobials, either as independent additives or as integrated monomeric components that are covalently incorporate into polymer networks to produce biomaterials with antibacterial, antifungal, antiviral and anti-matrix metalloproteinase activities [23]. Recently, a quaternary ammonium methacryloxy siliane molecule (QAMS; $\mathrm{C}_{44} \mathrm{H}_{90} \mathrm{ClNO}_{18} \mathrm{Si}_{5}$; CAS number 1566577-95-4) has been synthesized using silane-based sol-gel reaction [24]. One molecule of 3-(trimethoxysilyl)-propyldimethy loctadecyl ammonium chloride (SiQAC) and three molecules of 3methacryloxypropyltrimethoxysilane (3-MPTS) are attached to an anchoring unit-of tetraethoxysilane. The methacrylate groups derived from 3-MPTS are available for co-polymerization of methacrylate resin monomers. The long, lipophilic $\mathrm{C}_{18} \mathrm{H}_{37}$ alkyl chains derived from SiQAC are capable of puncturing bacteria cell walls and membranes, thereby providing a contact-active mechanism for eradicating biofilms that colonize the surface or interfacial gaps within the resin-based fillings [24,25]. In previous studies, incorporation of QAMS into orthodontic acrylic resin produced contact-killing antimicrobial activities against Streptococcus mutans, Actinomyces naeslundii and Candida albicans biofilms, without compromising the flexural strength and modulus of the polymethyl methacrylate [26].

Quaternary ammonium compounds possess anti-MMP [23,27$29]$ and anti-cathepsin $[23,30]$ potential. Hence, it is speculated that the combined antimicrobial and anti-proteolytic effects of QAMS would be effective in eliminating the detrimental effects caused by bacteria and proteolytic degradation of denuded collagen fibrils within the hybrid layers. Accordingly, different mass fractions of QAMS were incorporated into experimental adhesives in the present study, to test the hypotheses that: (1) adhesives containing QAMS possess antibacterial activities without adversely affecting their dentin bond strength, (2) QAMS has inhibitory effects on soluble MMP-9 and cathepsin K activities, and (3) hybrid layers treated with the most-optimal version of QAMS-containing adhesive are only minimally susceptible to degradation by endogenous dentin proteases.

\section{Materials and methods}

The QAMS utilized in the present work was purchased from KHG fiteBac Technology (Marietta, GA, USA) (Fig. 1A). Synthesis of the QAMS by sol-gel reaction had been described in a previous publication [24]. Eight-seven extracted sound human third molars were obtained according to a protocol approved by the Human Assurance Committee of Augusta University. All teeth except for those employed for cytotoxicity testing were stored in $0.9 \% \mathrm{NaCl}$ containing $0.02 \%$ sodium azide to prevent bacterial growth, and used within 3 months after retrieval.

\subsection{Bond strength to dentin}

Thirty teeth were used for bond strength testing. The roots of the teeth were removed $2-3 \mathrm{~mm}$ below the cementoenamel junction with a low-speed cutting saw (Isomet, Buehler Ltd., Lake Bluff, IL, USA) with water cooling. A flat mid-coronal dentin surface was exposed by cutting the occlusal enamel perpendicular to the longitudinal axis of each tooth. The exposed dentin was polished with 600 -grit wet silicon carbide paper for one minute to create a standardized smear layer.

Specimens were randomly allocated to five groups $(\mathrm{N}=6)$ according to the adhesives evaluated: experimental adhesives containing $0 \%, 2.5 \% 5 \%$ or $10 \%$ QAMS (designated as 0 -QAMS, 2.5-QAMS, 5-QAMS and 10-QAMS, respectively) and a commercial universal adhesive that was used as control (Clearfil Universal Bond (CF), Kuraray Noritake Dental Inc., Tokyo, Japan). The compositions of the experimental adhesives are listed in Table 1. The control universal adhesive CF was used in the etch-and-rinse mode. Each dentin surface was etched with $32 \%$ phosphoric acid (Uni-Etch, Bisco Inc., Schaumburg, IL, USA) for $15 \mathrm{~s}$, rinsed with deionized water for $15 \mathrm{~s}$ and gently air-dried for $5 \mathrm{~s}$ to keep the etched dentin visibly moist. Each adhesive was applied to the etched dentin and light-cured for $15 \mathrm{~s}$ using a light emission diode-curing unit. Resin composite build-ups (Z250, 3 M ESPE, St. Paul, MN, USA) were constructed using two 2-mm increments that were light-cured for $60 \mathrm{~s}$ each.

After storage in deionized water at $37{ }^{\circ} \mathrm{C}$ for $24 \mathrm{~h}$, the bonded specimens were sectioned vertically into $0.9 \mathrm{~mm}$-thick slabs with a low-speed cutting saw using water cooling. Five slabs from each tooth were sectioned into $0.9 \mathrm{~mm} \times 0.9 \mathrm{~mm}$ beams containing the resin-dentin interface in the center of the beam. The two longest beams from each slab were used for bond testing, resulting in 10 beams/tooth (i.e. 60 beams/group). Each beam was stressed to failure under tension with a universal testing machine using a crosshead speed of $1 \mathrm{~mm} / \mathrm{min}$. After testing, the cross-sectional area of each beam at the site of failure was measured for determining the tensile bond strength. Bond strength data derived from each tooth were averaged to generate the mean bond strength for that tooth. Statistical analysis was performed using the tooth as the statistical unit. After bond strength testing, the dentin side of each fractured beam was examined with a stereoscopical microscope at 40x magnification to identify the failure mode. Failure modes were classified as adhesive failure, mixed failure (failure extending 
A
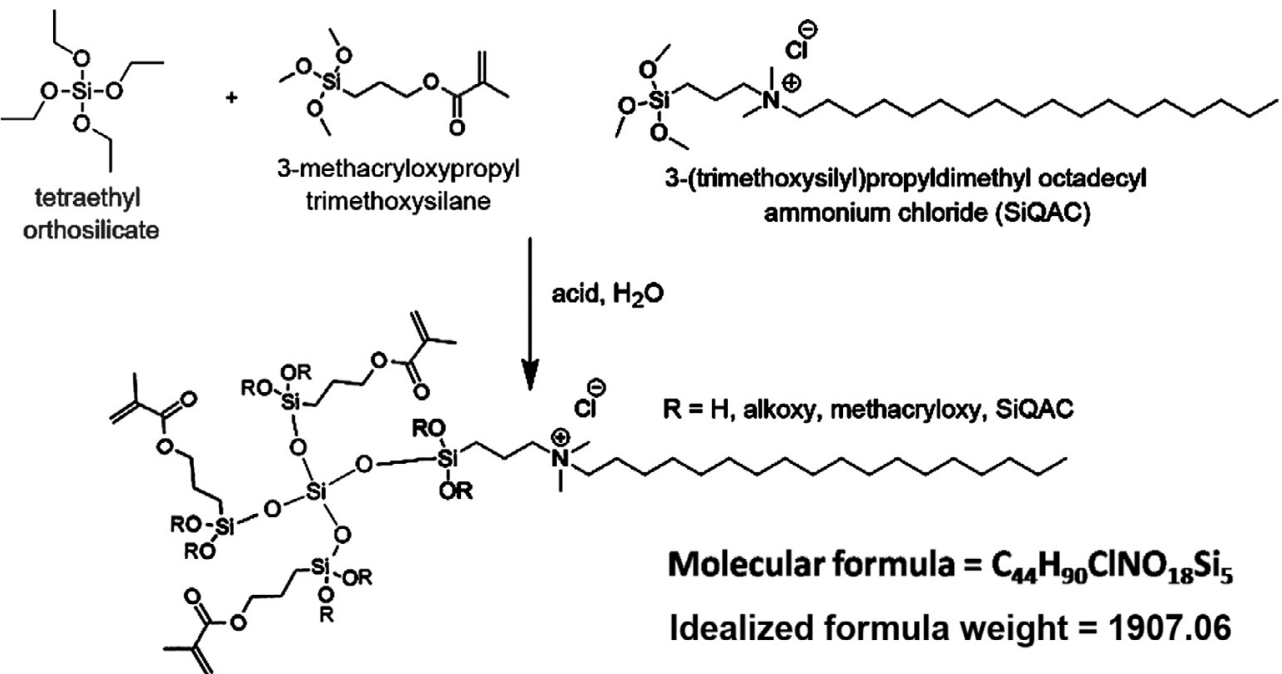

B

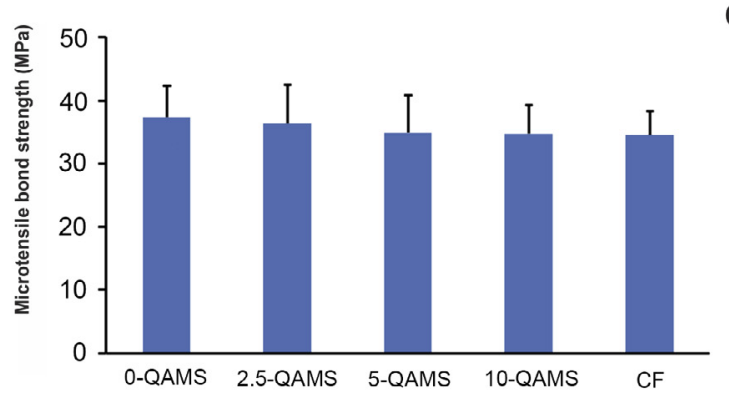

C

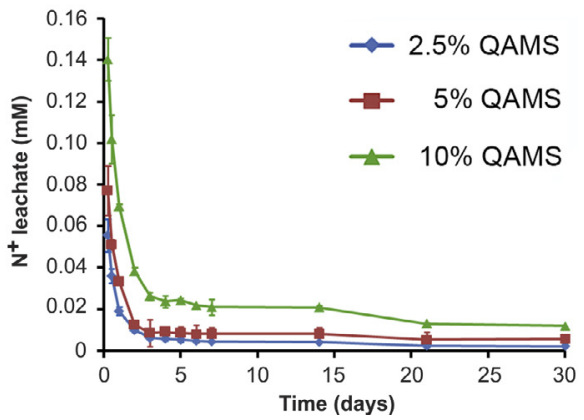

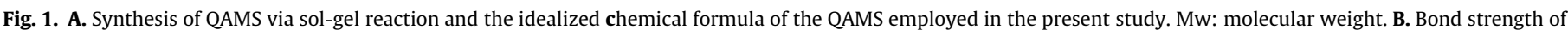
experimental and control adhesives to dentin. C. Leaching profiles of quaternary ammonium species from resin disks containing different concentrations of QAMS.

Table 1

Composition of experimental adhesives tested in the present study.

\begin{tabular}{lll}
\hline Adhesive & Composition & $\begin{array}{l}\text { Weight } \\
\text { percent }^{\dagger}\end{array}$ \\
\hline Experimental adhesives & Bis-GMA & $40.0 \%$ \\
& HEMA & $33.75 \%, 31.25 \%, 28.75 \%, 23.75 \%$ \\
& QAMS (0, 2.5, 5 and 10\%) & $0 \%, 2.5 \%, 5 \%, 10 \%$ \\
& C-MDP & $5.0 \%$ \\
& Camphorquinone & $1.0 \%$ \\
& Ethyl 4-dimethylaminobenzoate & $0.25 \%$ \\
Ethanol & $20 \%$
\end{tabular}

Abbreviations. Bis-GMA: bisphenol A glycidyl dimethacrylate; HEMA: 2-hydroxyethyl methacrylate; QAMS: quaternary ammonium methacryloxy silane; 10-MDP: 10-metha cryloxydecyl dihydrogen phosphate.

$\dagger$ Weight percentage of HEMA was reduced accordingly with the incorporation of different concentrations of QAMS.

into dentin or resin composite), cohesive failure in resin composite or cohesive failure in dentin.

\subsection{Leaching of quaternary ammonium species}

Experimental adhesives were placed into a mold and lightcured for $15 \mathrm{~s}$ to prepare $14.5 \mathrm{~mm}$ diameter and $1.5 \mathrm{~mm}$. thick resin disks. Disks from each of the three experimental adhesives (2.5-QAMS, 5-QAMS and 10-QAMS) were placed in a 24-well plate with $2 \mathrm{~mL}$ of deionized water in each well and agitated at $37{ }^{\circ} \mathrm{C}(\mathrm{N}$ $=8$ ) to collect eluents for bromophenol blue assay. Bromophenol blue is a dye which reacts with quaternary ammonium compounds to form a complex, with shift in the peak absorption wavelength from 590 to $603 \mathrm{~nm}$ [32]. A solution of $0.001 \mathrm{wt} \%$ bromophenol blue was prepared in deionized water and adjusted at $\mathrm{pH} 7.0$ with sodium carbonate. At $6 \mathrm{~h}, 12 \mathrm{~h}, 1-7,14,21$ and 30 days, a $0.5 \mathrm{~mL}$ aliquot of the leachate from each disk was collected, mixed with $1.5 \mathrm{~mL}$ of bromophenol blue and vortexed. Absorbance of the mixture was measured using a UV-VIS spectrometer (160A UV-VIS spectrometer, Shimadzu, Kyoto, Japan) at $603 \mathrm{~nm}$. The absorption values of complexes with known concentrations of SiQAC ranging from 0 to $.451 \mathrm{mM}$ were recorded to create a standard curve for the measurement of QAMS that leached from the resin disks after water sorption.

\subsection{Antibacterial activities}

\subsubsection{Bacteria culture}

Streptococcus mutans (ATCC 700610) and Actinomyces naeslundii (ATCC 12104) were used to examine the antibacterial activities of experimental adhesives containing different mass fractions of 
QAMS (0-QAMS, 2.5-QAMS and 5-QAMS). Streptococcus mutans was cultured aerobically in Brain Heart Infusion (BHI) broth at $37^{\circ} \mathrm{C}$. Actinomyces naeslundii was cultured in BHI under anaerobic conditions $\left(5 \% \mathrm{CO}_{2}, 90 \% \mathrm{~N}_{2}\right.$ and $\left.5 \% \mathrm{H}_{2}\right)$ at $37{ }^{\circ} \mathrm{C}$. The bacteria were grown overnight, harvested by centrifugation and washed three times with sterile phosphate-buffered saline (PBS). The bacteria were re-suspended in $\mathrm{BHI}$ and diluted to a final concentration of $1.0 \times 10^{7}$ colony-forming units (CFU)/mL. Bacteria density was determined using a spectrophotometer (Beckman Coulter, Inc., Indianapolis, IN, USA) at an optical density of $600 \mathrm{~nm}$.

\subsubsection{Agar diffusion test}

Bacterial suspensions $\left(1.0 \times 10^{7} \mathrm{CFU} / \mathrm{mL} ; 100 \mu \mathrm{L}\right)$ were plated on BHI agar plates using a spiral plater (easySpiral Plater, Topaz Inc., Cohasset, MA, USA). Resin disks (6.5 mm diameter; $1.5 \mathrm{~mm}$ thick) were prepared and placed on a bacteria-inoculated agar plate and incubated at $37^{\circ} \mathrm{C}$ for $24 \mathrm{~h}$. Inhibition areas surrounding the disks were measured with Image-Pro Plus 6.0 (Media Cybernetics, Inc., Silver Spring, MD, USA). The experiment was performed in triplicate.

\subsubsection{Live/dead bacterial staining}

Single-species biofilms grown on the surface of resin disks prepared from the three groups was stained using a LIVE/DEAD BacLight Bacterial Viability Kit (Molecular Probes, Invitrogen Corp., Carlsbad, CA, USA). The disks were incubated with bacteria at $37{ }^{\circ} \mathrm{C}$ for $24 \mathrm{~h}$, washed three times with PBS to remove unattached bacteria, and stained with $2.5 \mu \mathrm{M}$ SYTO 9 and propidium iodide [33]. A confocal laser scanning microscope (CLSM, LSM 780, Carl Zeiss, Oberkochen, Germany) was used to acquire images using a $20 \times$ objective lens, with the channels set at $480 / 500 \mathrm{~nm}$ (excitation/emission wavelengths) for SYTO 9, and 590/635 nm for propidium iodide. Each biofilm was scanned at five randomlyselected locations. Analyses of the three-dimensional architecture of the biofilms and the live/dead-stained bacteria biomass were performed using BiolmageL v2.1 (Faculty of Odontology, Malmo University, Malmo, Sweden) [34].

\subsubsection{Colony-forming unit (CFU) counts}

Streptococcus mutans and A. naeslundii biofilms were grown on the surface of disks for $24 \mathrm{~h}$. The disks were gently washed and transferred to polyethylene centrifuge tubes containing $1 \mathrm{~mL}$ of PBS. The tubes were vortexed and ultrasonicated to harvest bacteria. The harvested bacteria were plated on BHI agar plates and their viability was evaluated by CFU counting using a serial dilution method.

\subsubsection{Bacteria metabolism assay}

Bacteria dehydrogenase assay was performed with 3-(4,5-dime thylthiazol-2-yl)-2,5-diphenyltetrazolium bromide (MTT) assay to measure the metabolic activity of biofilms [35]. Disks with 24hour single-species biofilms were rinsed with PBS and incubated with MTT solution at $37{ }^{\circ} \mathrm{C}$ in $5 \% \mathrm{CO}_{2}$. The MTT dye is metabolized by live bacteria to form purple formazan. After one hour, dimethyl sulfoxide was added to solubilize the formazan crystals. Absorbance of the solubilized formazan was measured using the UVVIS spectrometer at $540 \mathrm{~nm}$.

\subsection{Inhibition of soluble rhMMP-9 and cathepsin $K$}

The inhibitory effect of QAMS on soluble rhMMP-9 was evaluated using purified recombinant human (rh) MMP-9 (AS-55576) and the Sensolyte Generic MMP assay kit (AS-72095) (Sensolyte, AnaSpec Inc., Fremont, CA, USA). The MMP assay kit contains an intact thiopeptolide that is cleaved by specific MMPs to release a sulfhydryl group. The sulfhydryl group forms colored 2-nitro-5thiobenzoic acid with Ellman's reagent.

A series of QAMS solutions (2.5\%,5\% and 10\%) was prepared. The thiopeptolide substrate solution was diluted to $0.2 \mathrm{mM}$ with assay buffer in a 1:50 vol ratio. In the experimental groups, each well contained $2 \mu \mathrm{L}$ of rhMMP-9 (19.6 ng/well), $10 \mu \mathrm{L}$ of potential MMP inhibitor and $50 \mu \mathrm{L}$ of thiopeptolide substrate solution. Additional assay buffer was added to generate $100 \mu \mathrm{L}$ per well.

The control groups included: (1) a positive control containing rhMMP-9 enzyme only without the potential anti-MMP agent; (2) an inhibitor control containing rhMMP-9 enzyme and $10 \mu \mathrm{L}$ of GM6001, a known MMP inhibitor; (3) a test compound control containing assay buffer and QAMS solutions at different concentrations; (4) a substrate control containing assay buffer. The reagents were mixed completely by shaking the plate for $30 \mathrm{~s}$. Readings were taken every $10 \mathrm{~min}$ for $60 \mathrm{~min}$ at $37^{\circ} \mathrm{C}$. Absorbance was measured at $412 \mathrm{~nm}$ using a 96-well plate reader. Background absorbance was determined from the "substrate control" wells and subtracted from the readings of the other wells containing the thiopeptolide substrate. The potencies of MMP-9 inhibition by MMP kit inhibitor (GM6001) and the three concentrations of QAMS were expressed as percentages of the adjusted absorbance of the "positive control". Inhibition of the MMP (\%) was calculated as 1 - $\left([\mathrm{A}]_{\text {test compound group }}-[\mathrm{A}]_{\text {test compound control }}\right) /\left([\mathrm{A}]_{\text {positive control }}-\right.$

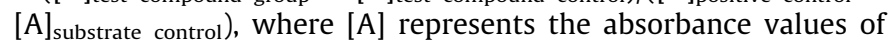
the wells. For each specimen, the mean absorbance value obtained from six wells ran in parallel was calculated.

The effect of QAMS on soluble cathepsin K was measured using Sensolyte 520 cathepsin K assay kits (AS-72171) (AnaSpec Inc.). The kit contains a QXL $^{\mathrm{TM}}$ 520/Hilyte Fluor ${ }^{\mathrm{TM}} 488$ FRET peptide substrate for cathepsin $\mathrm{K}$. When the FRET substrate is cleaved by active cathepsin $\mathrm{K}$, it releases HiLyte Fluor ${ }^{\mathrm{TM}} 488 \mathrm{~m}$, the fluorescence of which may be detected at $490 / 520 \mathrm{~nm}$ using a microplate reader. Cathepsin $\mathrm{K}$ inhibitory assay was performed as described for the MMP-9 assays. The efficacy of QAMS in inhibiting cathepsin K was calculated as percentages of the adjusted absorbance of the "positive control". Each experiment was performed in sextuplicate.

\subsection{In-situ zymography}

Ten teeth from each of the 0-QAMS, 5-QAMS and CF adhesive groups were used for in-situ zymography of the resin-dentin interface. Three drops of each adhesive were mixed with one grain of tetramethylrhodamine B isothiocyanate (excitation/emission: 540/625 nm; MilliporeSigma, St. Louis, MO, USA) to create a homogeneous solution [36]. After bonding with the respective dyed adhesive, a 2-mm thick layer of flowable resin composite was placed and light-cured for $20 \mathrm{~s}$. After storage in deionized water at $37^{\circ} \mathrm{C}$ for $24 \mathrm{~h}$, the bonded specimens were sectioned vertically into $1 \mathrm{~mm}$-thick slabs to expose the resin-dentin interface.

Each bonded slab was attached to a microscope slide with cyanoacrylate adhesive and polished to $\sim 50 \mu \mathrm{m}$ thick. Because highly cross-linked dentin collagen takes a relatively long time to be degraded by MMPs, a self-quenched fluorescein-conjugated gelatin was used as the MMP substrate (E-12055, Molecular Probes, Eugene, OR, USA), for identification of sites of MMP activity within the hybrid layers. A $1.0 \mathrm{mg} / \mathrm{mL}$ stock solution of selfquenched fluorescein-conjugated gelatin was prepared by adding $1.0 \mathrm{~mL}$ deionized water to the vial containing the lyophilized gelatin. The gelatin stock solution and an anti-fading agent (Mounting Medium with DAPI H-1200, Vectashield, Vector Laboratories LTD, Cambridgeshire, UK) were diluted in a 1:1:8 ratio with dilution buffer ( $\mathrm{NaCl} 150 \mathrm{mM}, \mathrm{CaCl}_{2} 5 \mathrm{mM}$, Tris- $\mathrm{HCl} 50 \mathrm{mM}$, pH 8.0). Then, $50 \mu \mathrm{L}$ of the fluorescent gelatin solution was placed on top of each polished section and protected with a cover slip. The glass slides 
were incubated in the dark in a humidity chamber at $37^{\circ} \mathrm{C}$ for $48 \mathrm{~h}$.

Each specimen was visualized with a two-photon confocal laser scanning microscope (CLSM, LSM 780, Carl Zeiss, Thornwood, NY, USA) equipped with a 40x oil immersion objective lens. Image channels were set at 488/530 nm. Green fluorescence derived from de-quenched fluorescein was imaged with the red fluorescence released by the adhesive. Optical sections ( $85 \mu \mathrm{m}$ thick) were acquired from different focal planes of each specimen. Stacked images were processed with ZEN 2010 software (Carl Zeiss). Quantification of the hydrolyzed gelatin was calculated based on the value of relative green fluorescence, using the Image-Pro Plus 6.0 software.

\subsection{Transmission electron microscopy}

Three teeth from each of the 0-QAMS, 5-QAMS and CF adhesive groups were used for transmission electron microscopy (TEM). After bonding with the respective adhesive, a 2-mm thick layer of flowable resin composite was placed over the bonded dentin and light-cured for $20 \mathrm{~s}$. A $1 \mathrm{~mm}$-thick dentin slab was sectioned longitudinally from the center of each bonded tooth, and further trimmed to produce $2 \times 1 \times 2 \mathrm{~mm}$ sticks. The sticks were completely demineralized in $0.1 \mathrm{M}$ formic acid/sodium formate, fixed with Karnovsky's fixative and post-fixed in 1\% osmium tetroxide. The fixed specimens were dehydrated in an ascending ethanol series (50-100\%), immersed in propylene oxide as a transition medium and embedded in pure epoxy resin. Ninety nanometer thick sections were e stained with $2 \%$ uranyl acetate and Reynold's lead citrate and examined a TEM (JEM-1230, JEOL, Tokyo, Japan) at $110 \mathrm{kV}$.

\subsection{Water permeation through resin-dentin interface}

Five teeth from each of the 0-QAMS, 5-QAMS and CF adhesive groups were used for permeability evaluation. Each tooth was prepared into a tooth segment by making parallel cuts perpendicular to the longitudinal axis of each tooth. The first cut was made at the enamodentinal junction. The second cut was made $2 \mathrm{~mm}$ below the cementoenamel function. The occlusal bonding surface was polished with 600-grit silicon carbide paper to remove traces of residual enamel. The remaining dentin thickness of each tooth was controlled to $2.5 \pm 0.1 \mathrm{~mm}$ from the deepest pulpal horn. Tooth segments with exposed pulp horns were discarded.

A double fluorescence technique was employed to enable the adhesive and the water that permeated the resin-dentin interface to be detected simultaneously with CLSM. One microliter of a yellow fluorescent dye (Alexa Fluor $^{\mathrm{TM}}$ 532, excitation/emission: 532/553 nm; ThermoFisher Scientific, Waltham, MA, USA) was dissolved in 3 drops of the respective adhesive and mixed homogeneously in the dark. The dentin segment to be bonded were attached to a perforated Plexiglass block using cyanoacrylate adhesive. The assembly was connected to a polyethylene tubing via an 18-gauge stainless steel tube. The polyethylene tubing was attached to a column of $0.1 \%$ blue fluorescent dye solution (Alexa Fluor $^{\mathrm{TM}} 405,401 / 421 \mathrm{~nm}$ ) [37] oriented $20 \mathrm{~cm}$ above the Plexiglass block to simulate the pulpal pressure present in non-inflamed dental pulps. Water pressure was delivered to the resin-dentin interface through the dentin tubules during bonding. The set-up was left in the dark for $4 \mathrm{~h}$ to enable water to continue permeate the bonded interface.

After removal of the bonded tooth segment from the Plexiglass block, a $1 \mathrm{~mm}$-thick bonded slab was sectioned from the center of the specimens. Each slab was attached to a microscope slide using cyanoacrylate adhesive and polished to approximately $50 \mu \mathrm{m}$ thick. Each specimen was examined with CLSM equipped using a 40x oil immersion objective lens. Blue fluorescence, indicative of the presence of transudated water within the resin-dentin interface, was imaged together with the yellow fluorescence released by the adhesive. Quantification of the blue fluorescence within and above the hybrid layer was calculated with Image-Pro Plus 6.0 to represent the relative permeability of the respective resindentin interface.

\subsection{Cytotoxicity}

Healthy human third molars designated for extraction were collected with the donors' written informed consent. The teeth were cleaned and cut perpendicular to the longitudinal axis of each tooth using sterile dental burs to expose the pulp chamber. Dental pulp tissues were gently removed using blunt non-cutting forceps, and dispersed in $2 \mathrm{mg} / \mathrm{mL}$ collagenase/dispase at $37{ }^{\circ} \mathrm{C}$ for $1.5 \mathrm{~h}$ to retrieve human dental pulp cells. The cells were grown in Dulbecco's Modified Eagle's Medium supplemented with $10 \%$ heatinactivated fetal bovine serum and $1 \%$ penicillin/streptomycin at $37{ }^{\circ} \mathrm{C}$ in a $95 \%$ relative humidity incubator supplemented with $5 \%$ $\mathrm{CO}_{2}$. The cultured cells were subsequently passaged after achieving $80 \%$ confluency. Fourth passage cells were used for the experiment.

Resin disks (6.5 mm diameter; $1.5 \mathrm{~mm}$ thick) were prepared from the 0-QAMS, 5-QAMS and CF adhesive groups. Sterile Teflon disks were used as the control. Disks in each group were incubated with culture medium ( 2 disks/mL; $\mathrm{N}=6$ ) at $37^{\circ} \mathrm{C}$ for 4 days to produce extract concentrates from the disks [24]. Each concentrate was diluted with fresh culture medium to $1: 10$ of its original concentration. The cells were cultured with the diluted media containing the respective resin eluents for 3 days before testing.

Cell viability was determined using MTT assay. The cells were seeded in a 96-well plate at a density of $1 \times 10^{4}$ cells per well and incubated in $100 \mu \mathrm{L}$ of medium per well for $24 \mathrm{~h}$. The medium was replaced with $100 \mu \mathrm{L}$ of fresh culture medium containing the respective resin eluents. After 3 days of incubation, MTT assay was performed and absorbance of solubilized formazan was measured at $490 \mathrm{~nm}$. The experiment as conducted in triplicate. Data were expressed as percentage viable cells compared with the Teflon negative control, which was taken to be $100 \%$ viable.

\subsection{Statistical analyses}

Data were expressed as means and standard deviations. For each parameter, data sets were examined for their normality (Shapiro-Wilk test) and equal variance assumptions (modified Levene test) prior to the use of parametric statistical methods. If those assumptions were not violated, the data sets were analyzed with one-factor analysis of variance (ANOVA) or one-factor repeated measures ANOVA, depending on the parameter tested. Post-hoc comparisons was conducted using Holm-Sidak procedures to identify statistical significance among groups. If the assumptions were violated, the data sets were nonlinearly transformed to satisfy those assumptions prior to performing the aforementioned statistical procedures. For all tests, statistical significance was set at $\alpha=0.05$.

\section{Results}

Tensile bond strengths of the four QAMS-containing experimental adhesives and the $\mathrm{CF}$ control adhesive are shown in Fig. 1B $(N=6)$. There was no significant difference among those groups ( $p>0.05$ ). The five groups had similar distribution of failure modes (Table 2). Generally, low bond strength values were associated with a higher tendency to fail within the adhesive [31]. Failure modes of the five adhesives consisted primarily of mixed failures, 
Table 2

Percentage distribution of failure modes of the five adhesives.

\begin{tabular}{|c|c|c|c|c|c|}
\hline \multirow[t]{2}{*}{ Failure mode } & \multicolumn{5}{|c|}{ Adhesives } \\
\hline & 0-QAMS & 2.5-QAMS & 5-QAMS & 10-QAMS & CF \\
\hline A & 11 & 10 & 11 & 13 & 14 \\
\hline $\mathrm{M}$ & 41 & 39 & 41 & 42 & 39 \\
\hline $\mathrm{CD}$ & 5 & 6 & 4 & 3 & 2 \\
\hline $\mathrm{CC}$ & 3 & 5 & 4 & 2 & 5 \\
\hline
\end{tabular}

Failure mode. A: adhesive; $\mathrm{CD}$, cohesive failure in dentin; $\mathrm{CC}$, cohesive failure in resin composite; M, mixed failure.

Table 3

Percentage of QAMS that leached out of experimental adhesive disks containing 2.5QAMS, 5-QAMS or 10-QAMS after immersion in deionized water, using bromophenol blue assay to quantify the amount of leached quaternary ammonium species.

\begin{tabular}{llll}
\hline Time (days) & $2.5 \%$ QAMS (\%) & $5 \%$ QAMS (\%) & $10 \%$ QAMS (\%) \\
\hline 0.25 & 1.07 & 1.18 & 1.70 \\
0.5 & 0.78 & 0.78 & 1.10 \\
1 & 0.51 & 0.53 & 0.58 \\
2 & 0.19 & 0.29 & 0.31 \\
3 & 0.14 & 0.19 & 0.20 \\
4 & 0.14 & 0.18 & 0.19 \\
5 & 0.13 & 0.16 & 0.18 \\
6 & 0.13 & 0.15 & 0.17 \\
7 & 0.12 & 0.14 & 0.16 \\
14 & 0.12 & 0.13 & 0.16 \\
21 & 0.08 & 0.08 & 0.10 \\
30 & 0.07 & 0.07 & 0.09 \\
\hline
\end{tabular}

with a small distribution of cohesive failure in resin composite and cohesive failure in dentin.

Fig. 1C shows release of quaternary ammonium moieties from polymerized resin disks containing different mass fractions of QAMS (2.5\%, 5\% and 10\%) from $6 \mathrm{~h}$ to 30 days. The percentages of quaternary ammonium species that leached out from resin disks containing $2.5 \%, 5 \%$ and $10 \%$ QAMS are shown in Table 3. There was a sharp decrease in $\mathrm{N}^{+}$species leaching from the adhesive disks after the first 2 days. Release at 2-30 days remained relatively stable for the three experimental groups. Adhesive disks containing $10 \%$ QAMS leached significantly more $\mathrm{N}^{+}$species than disks containing $2.5 \%$ or $5 \%$ QAMS for all testing periods $(\mathrm{p}<0.05)$. No significant difference was observed between the 2.5-QAMS and 5-QAMS groups ( $\mathrm{p}>0.05)$.

Because there was more leached quaternary ammonium species from resin disks containing 10\% QAMS, this concentration was not used for subsequently experiments. The results of agar diffusion tests for S. mutans and A. naeslundii are shown in Fig. 2 for resin disks containing $0 \%, 2.5 \%$ or $5 \%$ QAMS. For both bacteria species, resin disks containing no contact-killing QAMS monomer (0QAMS) did not produce inhibition areas. Bacteria growth could be clearly discerned under the transparent disks (Fig. 2A, C), indicating the polymerized adhesive possesses no inhibitory effect against the two bacterial species. Small inhibition areas were identified in the 2.5-QAMS group. Growth of bacteria colonies under the disks was slightly inhibited; bacterial colonies could not be seen beneath some parts of the disks. Resin disks containing $5 \%$ QAMS (5-QAMS) contained larger inhibition areas against both bacterial species. Small inhibition zones were identified around the disks, and growth of bacterial colonies was minimal underneath the disks. Quantitative evaluation of the areas of inhibition produced by the disks with $0 \%, 2.5 \%$ and $5 \%$ QAMS for each bacteria species are shown in Fig. 2B and D. The areas of inhibition produced by disks with $5 \%$ QAMS were $152.5 \pm 15.9 \mathrm{~mm}^{2}$ for $S$. mutans and $148.2 \pm 17.1 \mathrm{~mm}^{2}$ for $A$. naeslundii, respectively. These values were significantly higher than those in the 2.5-QAMS group $(\mathrm{p}<0.05)$, with average inhibitory areas of $59.3 \pm 10.6 \mathrm{~mm}^{2}$ for $S$. mutans and $31.6 \pm 12.3 \mathrm{~mm}^{2}$ for $A$. naeslundii, respectively.
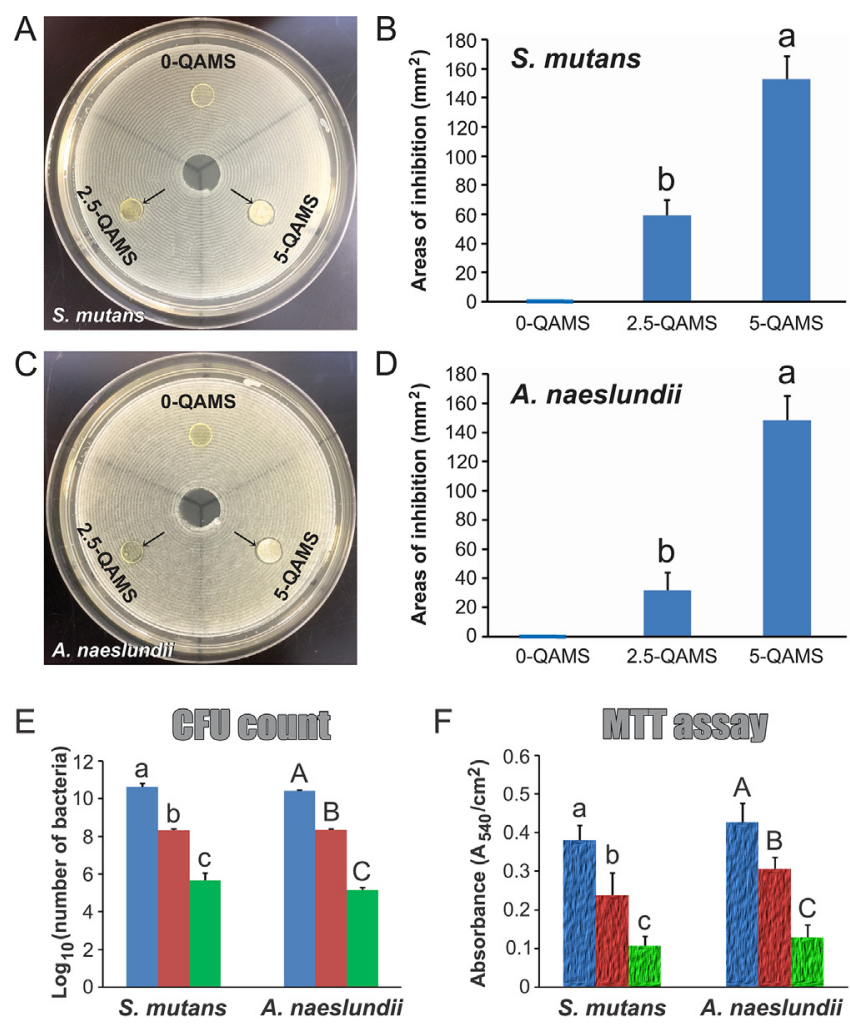

$\square$ 0-QAMS $\square$ 2.5-QAMS $\square$ 5-QAMS

0-QAMS 2.5-QAMS $\square$ 5-QAMS

Fig. 2. A. Agar diffusion assay (S. mutans). Arrows: inhibition areas. B. Bar chart showing areas of $S$. mutans inhibition in the three experimental adhesives. Data represent means \pm standard deviations. Columns identified with different letters are significantly different $(\mathrm{p}<0.05)$. C. Agar diffusion assay (A. naeslundii). Arrows: inhibition areas. D. Bar chart showing areas of $A$. naeslundii inhibition in the three experimental adhesives. Data represent means \pm standard deviations. Columns identified with different letters are significantly different $(\mathrm{p}<0.05)$. E. CFU counts. F. Bacteria MTT assay. Data in both charts represent means \pm standard deviations. For S. mutans, columns labeled with different low case letters are significantly different $(\mathrm{p}<0.05)$. For $A$. naeslundii, columns labeled with different upper case letters are significantly different $(\mathrm{p}<0.05)$.

Fig. 2E shows the CFU counts of resin disks after $24 \mathrm{~h}$ of $S$. mutans and $A$. naeslundii biofilm formation. For both $S$. mutans and A. naeslundii, resin disks containing $2.5 \%$ or $5 \%$ QAMS significantly reduced viable bacteria in the biofilms, compared with disks without QAMS ( $p<0.05$ ). Disks with 5\% QAMS were more efficient in reducing CFU counts compared with those containing 2.5\% QAMS ( $p<0.05)$. The results of biofilm dehydrogenase activities on QAMS-containing disks are shown in Fig. $2 F$. For both $S$. mutans and $A$. naeslundii, decrease in formazan absorbance with increase in QAMS concentrations could be identified. Resin disks containing 2.5\% QAMS had significantly reduction in biofilm metabolic activity compared with disks containing no QAMS ( $\mathrm{p}<0.05$ ). Disks with $5 \%$ QAMS had the lowest metabolic activity among these three groups.

Figs. $3 \mathrm{~A}$ and $4 \mathrm{~A}$ are representative CLSM images of the 24-hour three-dimensional architecture of $S$. mutans and $A$. naeslundii 
A

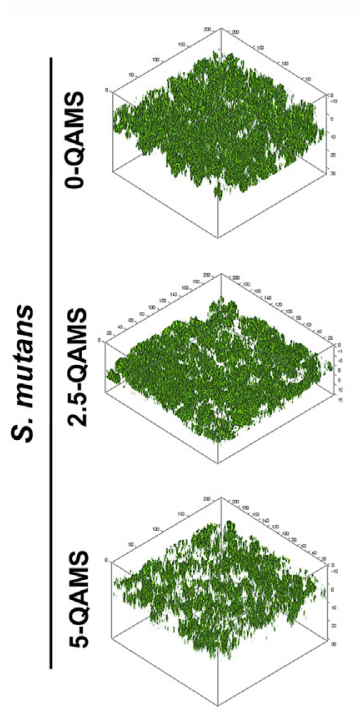

C

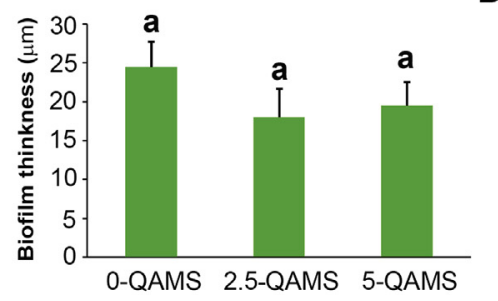

Dead
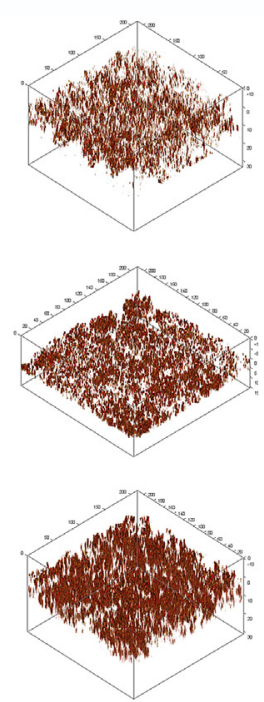

D

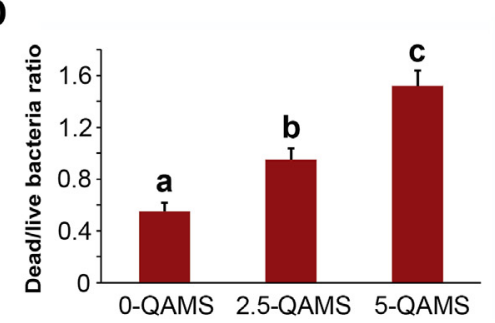

Merge
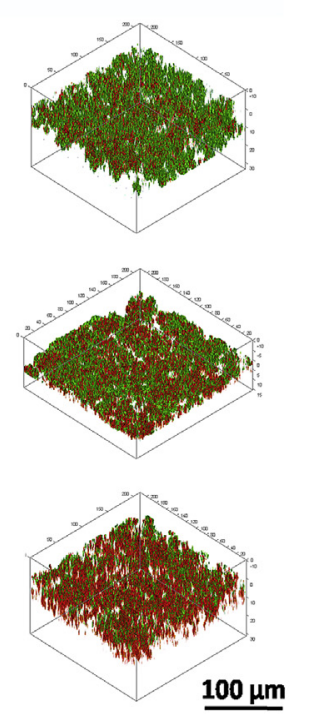

0-QAMS 2.5-QAMS 5-QAMS
B Biomass distribution along Z-stack
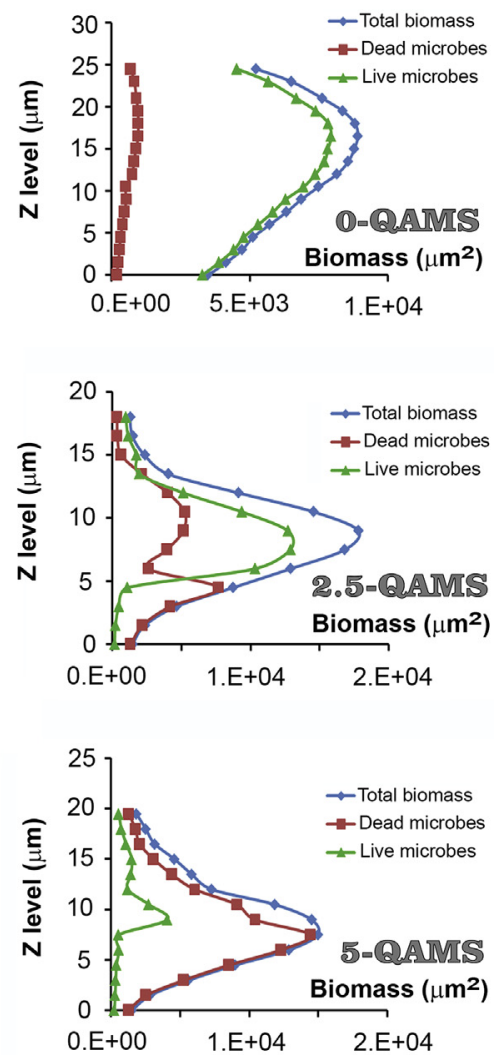

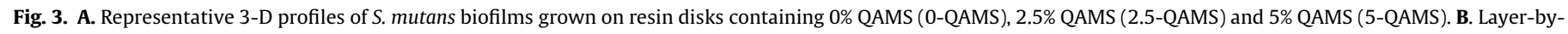

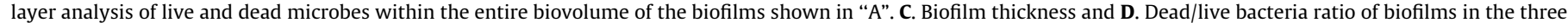
groups. Data in "C" and "D" are means \pm standard deviations. For each chart, columns labeled with different letters are significantly different ( $\mathrm{p}<0.05$ ).

biofilms stained with live/dead stains. Live bacteria were stained green while dead bacteria were stained red. Distributions of live and dead bacteria within the biomass of each layer of the Z-stack images are shown in Figs. 3B and 4B. Thickness of the biofilms in the three resin groups is summarized in Fig. $3 \mathrm{C}$ for $S$. mutans biofilms and Fig. 4C for A. naeslundii biofilms. No significant difference in the biofilm thickness among the three resin groups ( $p>0.05)$. For both $S$. mutans and A. naeslundii, biofilms grown on resin disks with $0 \%$ QAMS consisted of primarily live bacteria (Figs. 3B and 4B) and a low dead/live bacteria ratio (Figs. 3D and 4D). Biofilms grown on resin disks containing 2.5\% QAMS displayed a higher dead/live bacteria ratio compared with the 0 -QAMS group ( $\mathrm{p}<0.05)$. When the QAMS concentration increased from $2.5 \%$ to $5 \%$, the amounts of dead bacteria were significantly increased for both types of biofilms ( $p<0.05$ ). Compared with $0 \%$ and $2.5 \%$ groups, the dead/live bacteria ratio in the 5-QAMS group was much higher $(\mathrm{p}<0.05)$. For all QAMS-containing adhesives, dead bacteria were mainly detected at the base of the biofilms. Movies of stained 24-hour biofilms grown on the surface of resin disks derived from the 0-QAMS, 2.5-QAMS and 5-QAMS groups are shown respectively in Supplementary Videos 1-3 for S. mutans and Supplementary Videos 4-6 for A. naeslundii.

Inhibitory effects of QAMS on soluble rhMMP-9 and cathepsin K are shown in Fig. 5A. The relative percentages of rhMMP-9 and cathepsin $\mathrm{K}$ inhibition by the GM6001 (inhibitor control) were $95.8 \pm 4.7 \%$ and $77.5 \pm 2.0 \%$, respectively. Inhibition of rhMMP-9 by QAMS at concentrations higher than $5 \%$ was comparable with the kit inhibitor control ( $\mathrm{p}>0.05$ ). Although 2.5\% QAMS also inhibited rhMMP-9, its anti-MMP activity was significantly lower than $5 \%$ or $10 \%$ QAMS $(\mathrm{p}<0.05)$. The relative percentage of cathepsin
K inhibited by $5 \%$ QAMS was $72.0 \pm 3.5 \%$, which was not significantly different from 10\% QAMS or the inhibitor control ( $p>0.05$ ). By comparison, the anti-cathepsin activity of $2.5 \%$ QAMS was significantly lower than $5 \%$ QAMS or the inhibitor control $(p<0.05)$. Based on these results, 5\% QAMS appeared to be the minimum QAMS concentration required for optimal MMP-9 and cathepsin $\mathrm{K}$ inhibition. Accordingly, the experimental adhesive containing $5 \%$ QAMS was used for further validation using in-situ zymography of resin-dentin interfaces.

Fig. 5B summarizes the relative percentage areas of hybrid layers in the 0-QAMS, 5-QAMS and CF groups that exhibited fluorescence after coming in contact with the highly-quenched fluorescein-conjugated gelatin. Representative dual-channel CLSM images of the fluorescence in the three groups are shown in Fig. 5C. Dentin slabs prepared from specimens bonded with the QAMS-free experimental adhesive showed intense green fluorescence within the hybrid layers, reaching $79.6 \pm 7.4 \%$ fluorescence intensity. Green fluorescence was indicative of hydrolysis of the fluorescence-conjugated gelatin that into smaller peptides. Weaker green fluorescence was observed in hybrid layers created with the $5 \%$ QAMS-containing adhesive. The fluorescence value in this group $(19.5 \pm 6.9 \%)$ was significantly lower than that the QAMS-free experimental adhesive (0-QAMS) or the control CF adhesive ( $\mathrm{p}<$ $0.05)$.

Based on the results of the antibacterial and protease inhibition experiments, the experimental adhesive containing 5\% QAMS was selected as the optimized adhesive version for ultrastructural, water permeation and cytotoxicity evaluation. Ultrastructural features of the unfilled experimental adhesives were similar Irrespective of where QAMS was incorporated (Fig. 6A left) and were 
A

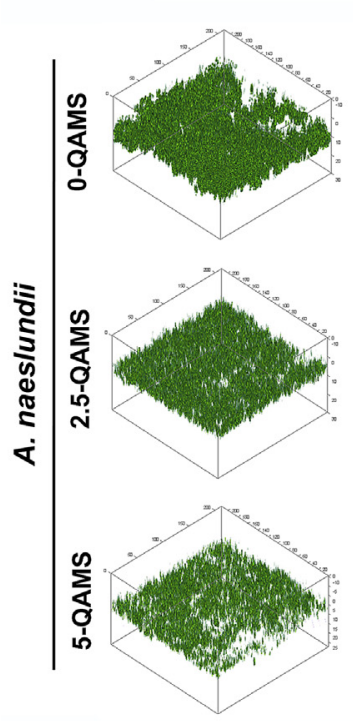

C

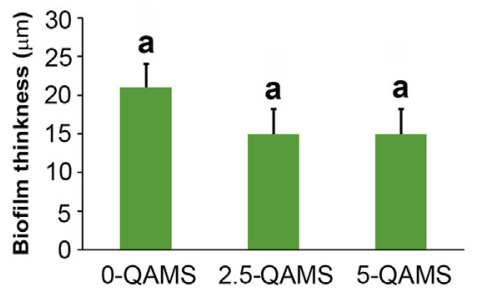

Dead

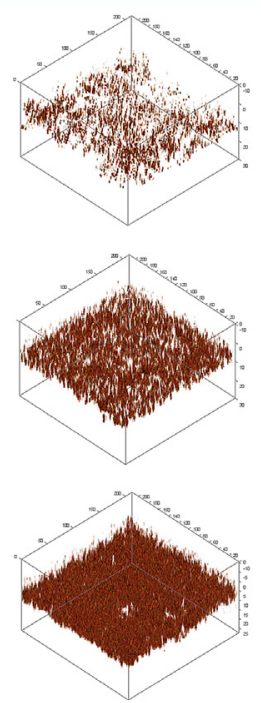

D

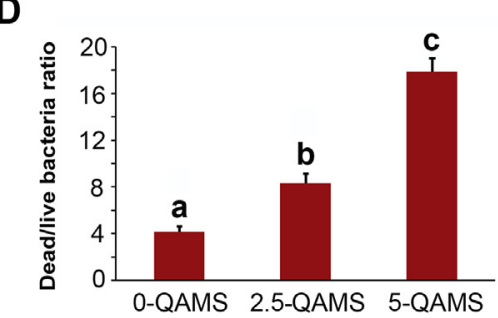

B Biomass distribution along Z-stack
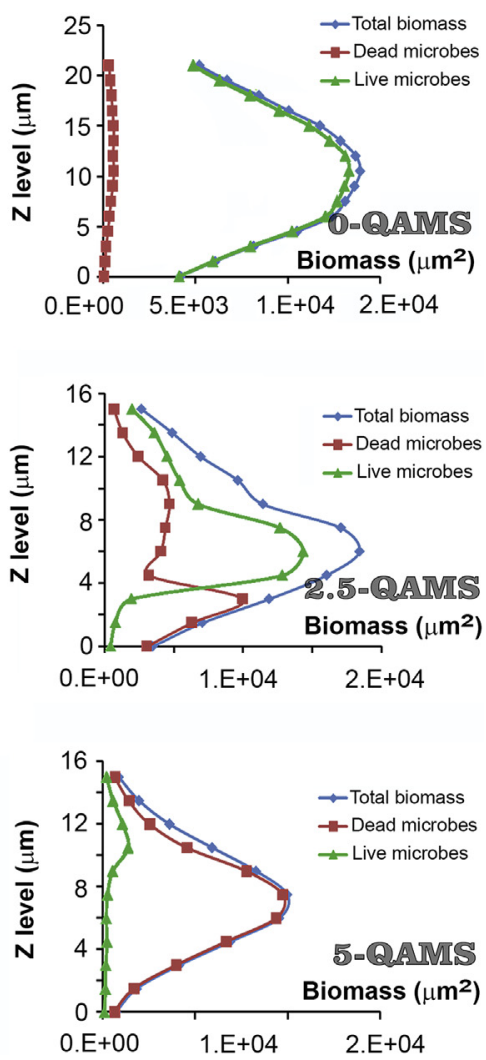

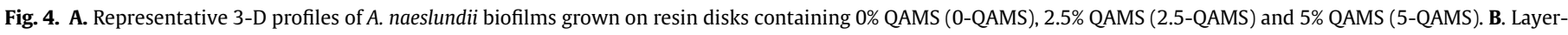

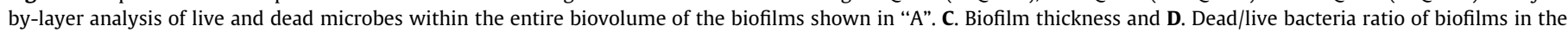
three groups. Data in "C" and "D" are means \pm standard deviations. For each chart, columns labeled with different letters are significantly different ( $\mathrm{p}<0.05$ ).

comparable with those observed in the control unfilled CF adhesive (Fig. 6A right). In stained, demineralized sections, the hybrid layers created by three adhesives were $5-8 \mu \mathrm{m}$ thick.

Representative CLSM images of the permeability of the resindentin interfaces created by the three adhesives (0-QAMS, 5QAMS and $(F)$ under simulated pulpal pressure conditions are shown in Fig. 6B. Areas occupied by the adhesives, including the dentin surface and dentinal tubules, exhibited strong yellow fluorescence. The hybrid layer, which contains a mixture of collagen and adhesive resin, exhibited weak yellow fluorescence. Sites of water permeation exhibited blue fluorescence. These sites included the dentinal tubules and their lateral branches, as well as the hybrid layer. Because all the adhesives examined are hydrophilic adhesives, water permeation could be identified within the hybrid layer. By superimposition of the fluorescence image over the differential interference contrast (DIC) image, water permeation was found to be limited to the hybrid layer only, No water channels or water bubbles could be identified within the adhesive and resin composite. The relative permeability of specimens bonded with the 5\% QAMScontaining adhesive was $52.0 \pm 9.9 \%$. Specimens bonded with the QAMS-free experimental adhesive or the CF adhesive had similar extents of water permeation within the hybrid layer, respectively resulting in $51.5 \pm 9.6 \%$ and $48.2 \pm 9.5 \%$ relative permeability. There was no significant difference among the three groups ( $p>0.05)$.

Compared with the Teflon negative control, human dental pulp cells that were exposed to eluents derived from 0-QAMS, 5-QAMS and CF resin disks were $90.9 \pm 10.0 \%, 78.2 \pm 6.2$ and $83.8 \pm 9.4 \%$, respectively. No significant difference was detected among the three groups $(\mathrm{p}>0.05)$.

\section{Discussion}

Adhesives intended for clinical use require considerable bond strength to dentin to prevent non-retentive restorations from dislodging during function. Inclusion of non-polymerizable antibacterial agents may alter the physical form of the polymerized resin matrix, resulting in reduction of physical properties [38]. In the present study, incorporation of $2.5 \%, 5 \%$ and $10 \%$ QAMS into the experimental resin blend had no adverse effect on the dentin bonding ability. The results are similar to a previous study that incorporated a different quaternary ammonium resin monomer into adhesive formulations [39]. The curing properties of an adhesive system is one of the key factors for obtaining strong bonding to dentin [40]. The photoinitiator-amine system utilized for the experimental adhesive formulations is a relatively simple system that does not include ternary catalysts for buffering the acidity of resin monomers during the application of self-cured composites [41], or prevent phase separation of hydrophilic from hydrophobic resin components in the presence of water moisture [42]. It would be interesting to compare the degree of monomer conversion in experimental adhesives that contain different amounts of QAMS, and examine the effect of different initiator/accelerator systems on nanoscopical phase separation of resin components in future studies.

Water sorption is responsible for hydrolysis and degradation of resin components. Sorption of water molecules into a polymerized resinous network provides a route for leaching of water-soluble unreacted resin monomers and salivary and bacterial enzymedegraded resin oligomers over time [43]. The chemical structure 
A

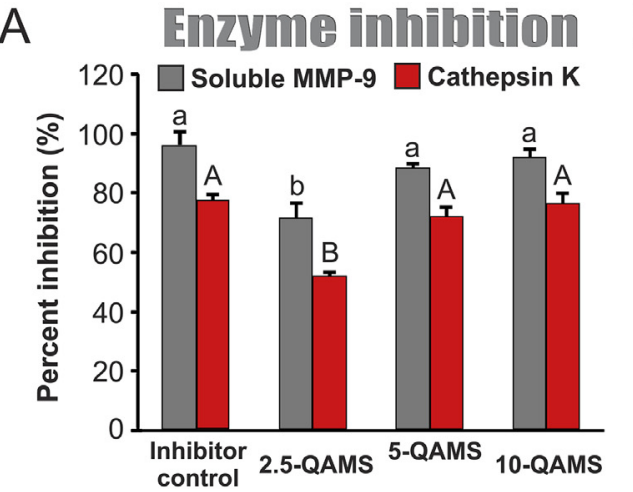

B In-situ zymography

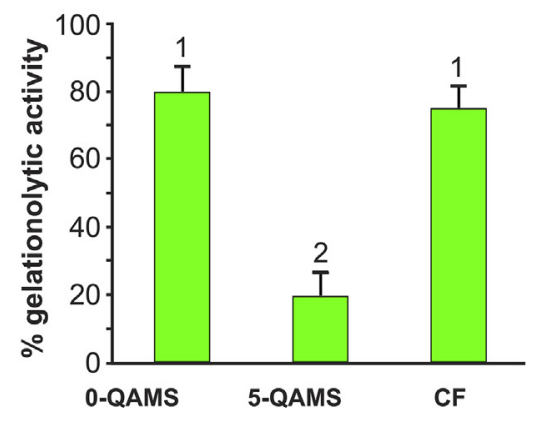

C

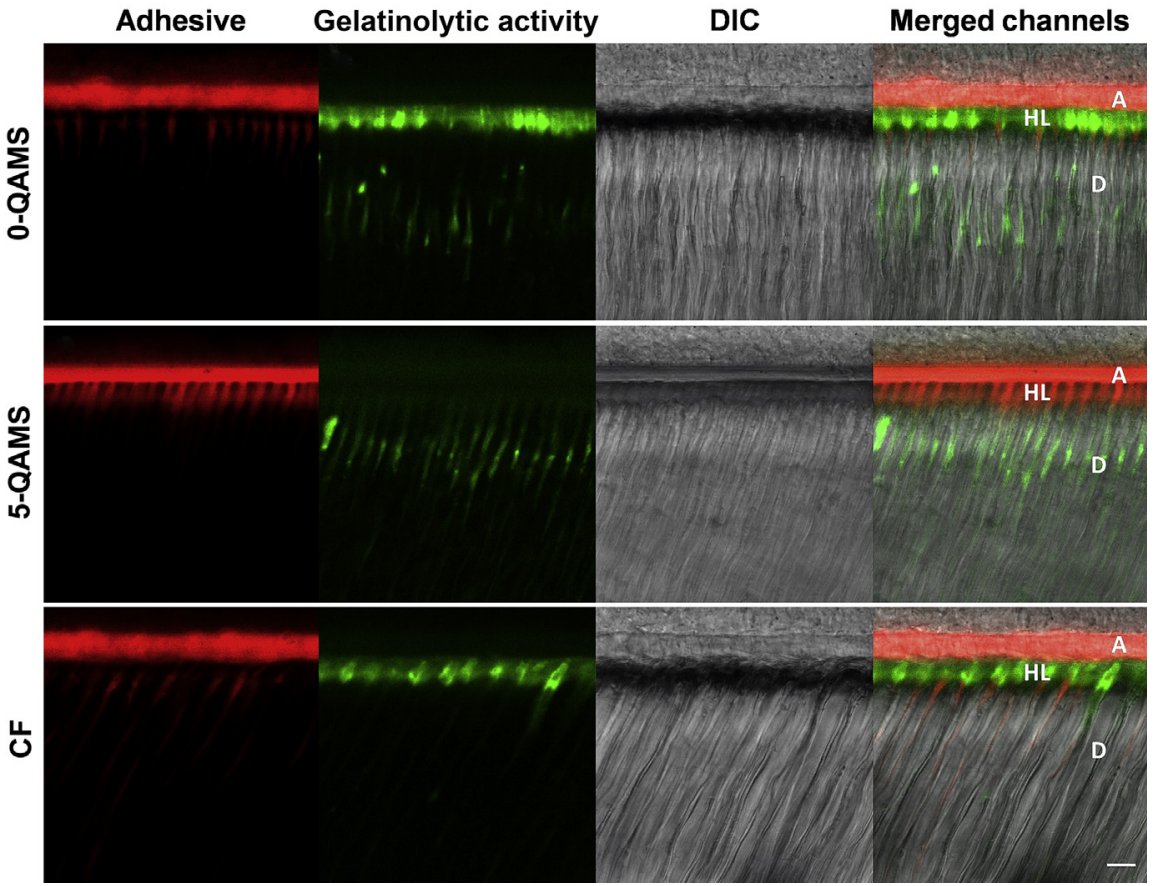

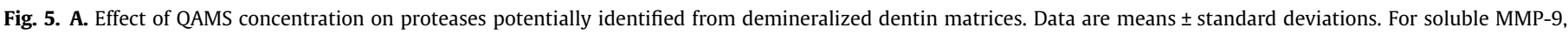

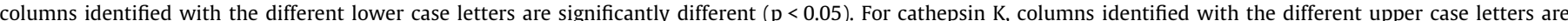

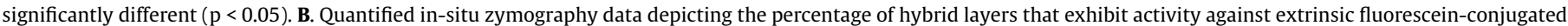

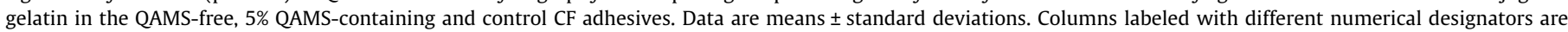

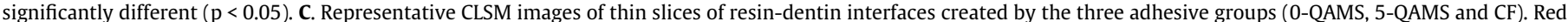

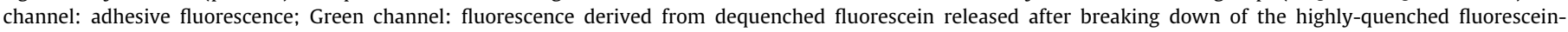

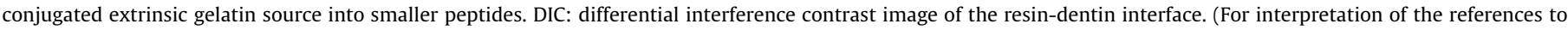
colour in this figure legend, the reader is referred to the web version of this article.)

of the QAS shown in Fig. 1A likely represents a statistical average based on the sol-gel condensation process, where some structures will have multiple QAS arms and others will have none. In addition, these siloxane structures are known to be quite sensitive to hydrolysis as well as exchange with alcohol in the presence of acidic catalysis [24]. The present adhesive formulation contains ethanol and MDP. This means the intended QAS structure may be inherently unstable within the solvated adhesive resin. Hence, incompletely co-polymerized QAMS within the polymer matrix would be expected to be released after aging in water or saliva. The bromophenol blue assay, commonly used in the textile industry for detecting leached quaternary ammonium salts from antimicrobial fabrics [44], was used to monitor the amount of quaternary ammonium moieties released from the QAMS-containing experimental adhesives. Results from the present study demonstrated continuous release of unreacted quaternary ammonium moieties from QAMS-containing resin disks into water during the first 30 days. Because QAMS is primarily co-polymerized with other methacrylate-based adhesive resin monomers, leaching was minuscule for formulations containing $\leq 5 \%$ QAMS, compared with antibacterial materials that are primary designed for diffusional kill that exhibit wide inhibition zones [45]. Incorporation of $10 \%$ QAMS significantly increased the amount of leachable $\mathrm{N}+$ species. Accordingly, the experimental adhesive containing 10\% QAMS was not further examined and only formulations containing $2.5 \%$ and 5\% QAMS were used for the antibacterial experiments.

Streptococcus mutans and A. naeslundii are cariogenic oral pathogens associated with secondary caries [46]. These microbes were used to evaluate the antibacterial properties of QAMS-containing experimental adhesives. Quaternary ammonium compounds is an important class of antibacterial agents that possess contactactive killing properties [23]. Unlike agents that function via a biocide-releasing approach, such as chlorhexidine, triclosan or silver nanoparticles, quaternary ammonium compounds are more environmental friendly and is less likely to contribute to the development of bacterial resistance [47]. Quaternary ammonium 


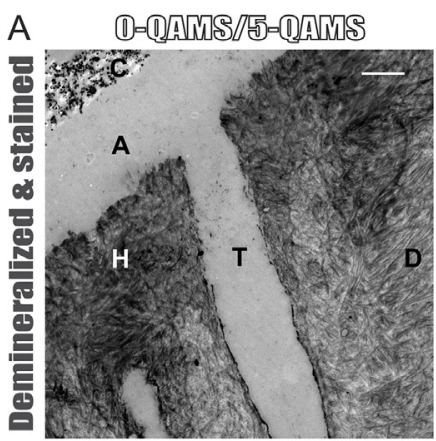

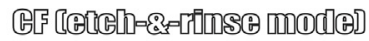

B
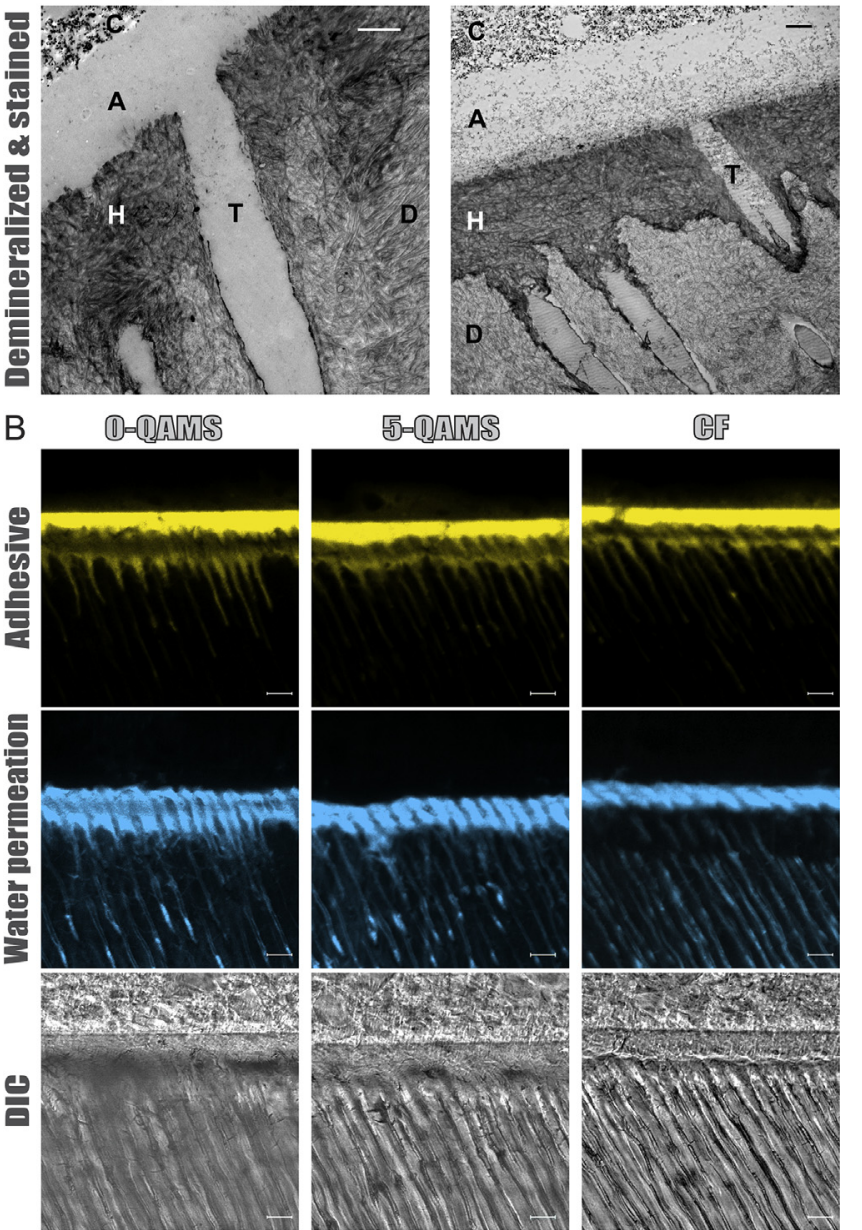

战
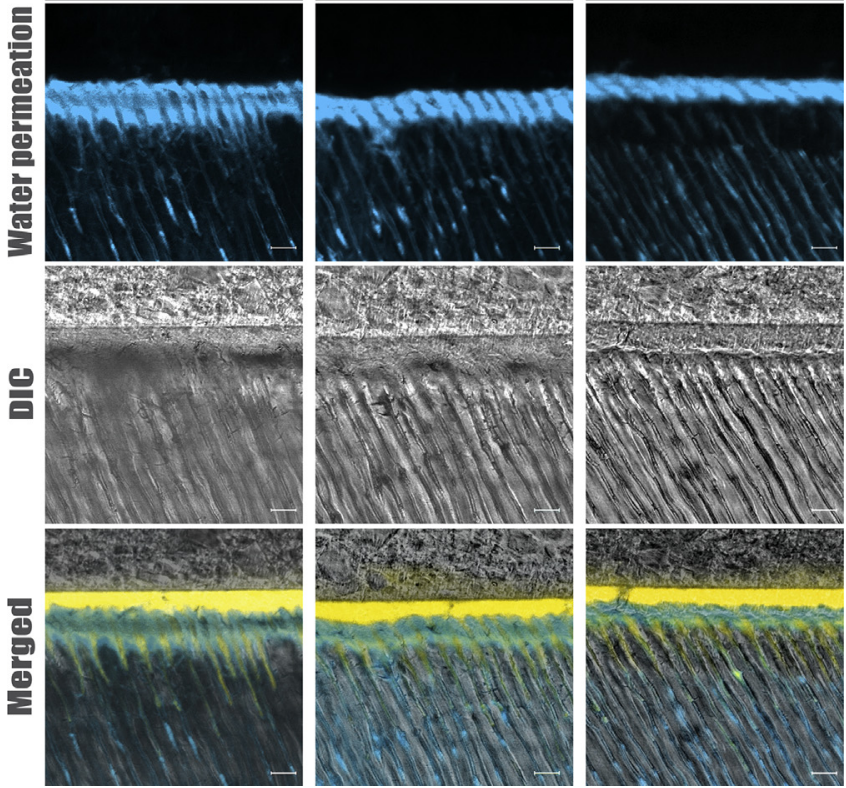

Fig. 6. A. Transmission electron microscopy images of resin-dentin interfaces created by the QAMS-free or 5\% QAMS-containing adhesive (left) and the control CF adhesive (right). Bars $=2 \mu \mathrm{m}$. Because the images were similar for the 0-QAMS and 5-QAMS groups, only the image 5-QAMS image is shown. C - resin composite; A adhesive; H - hybrid layer; T - dentinal tubule; D: demineralized intertubular dentin. B. Representative CLSM images depicting the water permeability along resin-dentin interfaces in the three adhesive groups (0-QAMS, 5-QAMS and CF). Bars $=10 \mu \mathrm{m}$. Yellow channel: adhesive fluorescence; Blue channel: fluorescentdye containing water that permeated the resin-dentin interface. DIC: differential interference contrast image of the resin-dentin interface. (For interpretation of the references to colour in this figure legend, the reader is referred to the web version of this article.)

compounds can be bound onto substrate surfaces to render those surfaces contact-active [23]. With a long, lipophilic $\mathrm{C}_{18} \mathrm{H}_{37}$ alkyl chain derived from SiQAC, QAMS achieves contact-killing by penetrating the cell walls and cell membranes of adherent bacteria [26]. When bacteria with negatively-charged cell wall come into contact with the positively-charged $\left(\mathrm{N}^{+}\right)$surface of the QAMS-containing adhesive through electrostatic attraction, they are punctured by the long alkyl chains, causing leakage of cytoplasmic components and subsequently cell death. The results of agar diffusion test further confirmed this contact-killing mechanism. Antimicrobial agents that eradicate microbes via diffusion usually produce relatively large inhibition zones around the tested material [45].
Inhibition zones produced by the QAMS-containing adhesives were miniscule. In contrast, agar plate areas that were in direct contact with the 5\% QAMS-containing resin disks did not support the growth of bacterial colonies. Compared with resin disks containing $5 \%$ QAMS, the amount of QAMS in the 2.5\% QAMS-containing disks was inadequate to exhibit strong antibacterial effects, with incomplete contact killing of the bacteria colonies underneath the transparent resin disks. The small peripheral inhibition zones around the 5\% QAMS-containing resin disks are indicative of slight leaching of QAMS from the polymerized disks, confirming the results of bromophenol blue assay.

Three-dimensional reconstruction of CLSM images with live/ dead staining enables distribution of live and dead bacteria to be identified from different vertical planes of a biofilm. The present data showed that the QAMS-containing adhesives killed S. mutans and $A$. naeslundii in a dose-dependent manner, predominantly via contact-killing. Nevertheless, dead bacteria was not only confined to the bottom of the biofilms that was in contact with the adhesive surface (Fig. 3B, 4B and Supplementary Videos). A gradual reduction in dead microbial biomass was also observed with increasing distance from the base of the biofilms. This observation is similar to previous in vitro and in vivo reports of dead microbial profiles generated by QAMS-containing orthodontic polymethyl methacrylate resins $[25,48]$. One possible explanation for this phenomenon is the leaching of non-polymerized monomer components from the adhesive substrate, which may have resulted in diffusional-killing of bacteria within the bulk of biofilms. Another reason is the regulation effect of the quorum-sensing system within the bacteria consortium in a biofilm. Programed cell death of bacteria that reside superior to the base of the biofilm may occur via the release of pheromones or bacteriocins by incompetent bacteria at the bottom of biofilm in response to environmental stresses [49,50]. This coordinated altruistic cell death in the biofilm community enables elimination of non-competent members of the community, preservation of nutrients, as well as release of genomic materials into the biofilm matrix (i.e. extracellular DNA), to support survival of the more competent members of that community [51]. Counting of CFUs and MTT metabolic assays were performed to complement the CLSM data. The results confirmed that the reduction in viable bacteria was dependent upon the concentration of QAMS incorporated into the adhesive. Based on these antibacterial results and the bond strength data, the first hypothesis that "adhesives containing QAMS possess antibacterial activities without adversely affecting their dentin bond strength" is validated.

Demineralized collagen matrices of dentin act as scaffolds for resin infiltration during dentin bonding. Nevertheless, the dentin organic matrix contains soluble and matrix-bound endogenous proteolytic enzymes, including MMPs and cysteine cathepsins [52]. These proteolytic enzymes are responsible for degradation of exposed collagen fibrils within the hybrid layers. Activation of these proteases is perceived contemporarily as an important contributor to the poor durability of resin-dentin bonds [53]. Matrix metalloproteinase are a family of $\mathrm{Zn}$ - and Ca-dependent enzymes [54]. When activated by acid-etchants during dentin bonding, some of the MMPs, specifically MMP-2, MMP-8 and MMP-9, are responsible for degradation of extracellular matrix components [53]. Although dentin type I collagen is highly cross-linked [55], they are still susceptible to the degradation by dentin-specific MMPs [52]. Being the only cysteine cathepsin identified from mineralized dentin, cathepsin $\mathrm{K}$ cleaves the telopeptides into slightly shorter fragments than what is achieved by MMPs [56]. In the present work, MMP-9 and cathepsin K were used for evaluating the potent inhibitory effect of QAMS using Sensolyte assay kits. The results of the quantitative assay showed that the extent of rhMMP-9 and cathepsin $\mathrm{K}$ inhibition was proportional to QAMS concentrations. Hence, the second hypothesis that "QAMS has inhi- 
bitory effects on soluble MMP-9 and cathepsin K activities" is validated.

In-situ zymography is a simple, rapid laboratory technique for localization of protease activities in tissue sections [57]. This method enables screening of the relative proteolytic activities directly within dentin hybrid layers [58]. The present work shows weak fluorescence in hybrid layers produced using the experimental adhesive containing 5\% QAMS. In contrast, specimens bonded with the QAMS-free adhesive exhibited intense green fluorescence within the hybrid layers after incubation of the quenched fluorescein-conjugated gelatin for $48 \mathrm{~h}$, with 4 -fold increase in gelatinolytic activity. Hence, the third hypothesis that "hybrid layers treated with the most-optimal version of QAMS-containing adhesive are only minimally susceptible to degradation by endogenous dentin proteases" is validated.

Because of its cationic quaternary ammonium group, the QAMS may bind electrostatically to the negatively-charged carboxylic groups in the collagen fibrils and noncollagenous proteins of demineralized dentin [59]. This non-specific binding may affect the activation of MMPs, making them unable to accept the complementary peptide sequence for collagen. The catalytic domains of MMPs contain cysteine-rich sites, including a glutamic acid residue with negative charge [53]. The cationic QAMS may alter the configuration of the catalytic site of the MMPs via electrostatic binding to the negatively-charge glutamic acid residues, sterically blocking the active site and inhibiting MMP activities [60]. The catalytic sites of cysteine cathepsins contain cysteine, histidine and aspartame residues. Cysteine forms a catalytic thiolate-imidazolium ion pair that acts as a nucleophile for attack on the carbonyl carbon atom of the peptide bond [30]. It is possible that the cationic QAMS binds electrostatically to the cathepsin active site to inhibit its activity. These factors may have contributed to protecting the demineralized dentin collagen matrix from enzymatic degradation over time.

In the authors' previous work [59], the inhibition effect of quaternary ammonium silane on MMP activities had already been tested and confirmed using MMP-2 enzyme-linked immunosorbent assay. Hence, the inhibition effects of the QAMS on dentin MMP activities were evaluated in the present study using another MMP (viz. MMP-9). With respect to the dentin-bound cysteine cathepsins, both cathepsin B and cathepsin $\mathrm{K}$ are present in sound and carious dentin. However, the authors had been investigating predominantly inhibition of dentin cathepsin $\mathrm{K}$ by quaternary ammonium compounds [30] because of the ability of this cathepsin to release the C-terminal telopeptide fragment. Thus, MMP-9 and cathepsin $\mathrm{K}$ were chosen as representative examples of MMP and cysteine cathepsin inhibition in the present work.

The bonded dentin interface should be properly sealed to protect itself from water permeation. Nevertheless, intrapulpal pressure enables constant replenishment of intrinsic water from the pulp chamber to the dentin and contributes to the poor durability of resin-dentin bonds. In the present study, a double-fluorescence technique was used to examine interfacial permeability after bonding. In the presence of simulated pulpal pressure, zones of interfacial permeability could be identified throughout the hybrid layer in all the three adhesives examined. The results indicate that the goal of achieving a fluid-tight seal has not been perfectly accomplished. This issue is characteristic of contemporary adhesives containing hydrophilic resin monomers and should be duly addressed in the future for optimizing resin-dentin bond durability.

\section{Conclusion}

Within the limitations of the present short-term in vitro study, it may be concluded that incorporation of $5 \%$ QAMS into the experimental adhesive formulation achieves concomitant antibacterial and anti-proteolytic effects without compromising dentin bond strength. The 5\% QAMS-containing bioactive adhesive with low cytotoxicity may play a role in eliminating the secondary caries and preventing hybrid layer degradation. Although a commercially available 2-methacryloyloxydodecyl pyridinium bromide containing adhesive (Clearfil Protect Bond, Kuraray Noritake Dental Inc.) also possesses these activities, it is intended for use in the self-etching mode instead of the etch-and-rinse mode. Further long-term aging studies and animal studies are required to support the potential clinical application of the presently developed bioactive QAMS-containing etch-and-rinse adhesive.

\section{Acknowledgments}

This work was supported by National High Technology Research and Development Program of China grant 2015AA020942, National Nature Science Foundation of China grant 81400555 and Natural Science Basic Research Plan in Shaanxi Province of China grant 2015JM8383 (Li-na Niu, The Fourth Military Medical University). The authors declare no potential conflicts of interest with respect to the authorship and/or publication of this work.

\section{Appendix A. Supplementary data}

Supplementary data associated with this article can be found, in the online version, at https://doi.org/10.1016/j.actbio.2018.06.008.

\section{References}

[1] J.L. Ferracane, Resin composite-state of the art, Dent. Mater. 27 (2011) 29-38.

[2] I. Nedeljkovic, W. Teughels, J. De Munck, B. Van Meerbeek, K.L. Van Landuyt, Is secondary caries with composites a material-based problem?, Dent Mater. 31 (2015) e247-277.

[3] V. Thompson, R.G. Craig, F.A. Curro, W.S. Green, J.A. Ship, Treatment of deep carious lesions by complete excavation or partial removal: a critical review, J. Am. Dent. Assoc. 139 (2008) 705-712.

[4] L.J. Walsh, A.M. Brostek, Minimum intervention dentistry principles and objectives, Aust. Dent. J. 58 (Suppl 1) (2013) 3-16.

[5] B. Nyvad, M. Kilian, Microbiology of the early colonization of human enamel and root surfaces in vivo, Scand. J. Dent Res. 95 (1987) 369-380.

[6] M. Turkun, L.S. Turkun, Z. Ergucu, M. Ates, Is an antibacterial adhesive system more effective than cavity disinfectants?, Am J. Dent. 19 (2006) 166-170.

[7] R. Hickel, J. Manhart, Longevity of restorations in posterior teeth and reasons for failure, J. Adhes. Dent. 3 (2001) 45-64.

[8] E.A. Kidd, O. Fejerskov, What constitutes dental caries? Histopathology of carious enamel and dentin related to the action of cariogenic biofilms, J. Dent. Res. 83 (2004). Spec No C:C35-38.

[9] L. Breschi, A. Mazzoni, A. Ruggeri, M. Cadenaro, R. Di Lenarda, E. De Stefano, Dorigo, Dental adhesion review: aging and stability of the bonded interface, Dent. Mater. 24 (2008) 90-101.

[10] Y. Liu, L. Tjäderhane, L. Breschi, A. Mazzoni, N. Li, J. Mao, D.H. Pashey, F.R. Tay, Limitations in bonding to dentin and experimental strategies to prevent bond degradation, J. Dent. Res. 90 (2011) 953-968.

[11] L. Tjäderhane, F.D. Nascimento, L. Breschi, A. Mazzoni, I.L. Tersariol, S. Geraldeli, A. Tezvergil-Mutluay, M. Carrilho, R.M. Carvalho, F.R. Tay, D.H. Pashley, Strategies to prevent hydrolytic degradation of the hybrid layer-a review, Dent. Mater. 29 (2013) 999-1011.

[12] M.R. Carrilho, R.M. Carvalho, M.F. de Goes, V. di Hipolito, S. Geraldeli, F.R. Tay, D.H. Pashley, L. Tjäderhane, Chlorhexidine preserves dentin bond in vitro, J. Dent. Res. 86 (2007) 90-94.

[13] F.R. Tay, D.H. Pashley, R.J. Loushine, R.N. Weller, F. Monticelli, R. Osorio, Selfetching adhesives increase collagenolytic activity in radicular dentin, J. Endod. 32 (2006) 862-868.

[14] Y. Nishitani, M. Yoshiyama, B. Wadgaonkar, L. Breschi, F. Mannello, A. Mazzoni, R.M. Carvalho, L. Tjäderhane, F.R. Tay, D.H. Pashley, Activation of gelatinolytic/collagenolytic activity in dentin by self-etching adhesives, Eur. J. Oral Sci. 114 (2006) 160-166.

[15] A. Mazzoni, P. Scaffa, M. Carrilho, L. Tjäderhane, R. Di Lenarda, A. Polimeni, A. Tezvergil-Mutluay, F.R. Tay, D.H. Pashley, L. Breschi, Effects of etch-and-rinse and self-etch adhesives on dentin MMP-2 and MMP-9, J Dent Res. 92 (2013) 82-86.

[16] A. Frassetto, L. Breschi, G. Turco, G. Marchesi, R. Di Lenarda, F.R. Tay, D.H. Pashley, M. Cadenaro, Mechanisms of degradation of the hybrid layer in 
adhesive dentistry and therapeutic agents to improve bond durability - a literature review, Dent. Mater. 32 (2016) 41-53.

[17] M. Bourbia, D. Ma, D.G. Cvitkovitch, J.P Santerre, Y. Finer, Cariogenic bacteria degrade dental resin composites and adhesives, J Dent Res. 92 (2013) 989-994.

[18] B. Huang, W. Siqueira, D.G. Cvitkovitch, Y. Finer, Esterase from a cariogenic bacterium hydrolyzes dental resins, Acta Biomater. 71 (2018) 330-338.

[19] S. Twetman, Antimicrobials in future caries control? A review with special reference to chlorhexidine treatment, Caries Res. 38 (2004) 223-229.

[20] R. Gendron, D. Grenier, T. Sorsa, D. Mayrand, Inhibition of the activities of matrix metalloproteinases 2, 8, and 9 by chlorhexidine, Clin. Diagn. Lab. Immunol. 6 (1999) 437-439.

[21] L. Cheng, M.D. Weir, H.H. Xu, A.M. Kraigsley, N.J. Lin, S. Lin-Gibson, Antibacterial and physical properties of calcium-phosphate and calciumfluoride nanocomposites with chlorhexidine, Dent. Mater. 28 (2012) 573-583.

[22] F.T. Sadek, R.R. Braga, A. Muench, Y. Liu, D.H. Pashley, F.R. Tay, Ethanol wetbonding challenges current anti-degradation strategy, J. Dent. Res. 89 (2010) 1499-1504.

[23] J. Yang, L.-N. Niu, S. Ma, J. Li, F.R. Tay, J.-H. Chen, Quaternary ammonium-based biomedical materials: State-of-the-art, toxicological aspects and antimicrobial resistance, Prog. Polym. Sci. 71 (2017) 53-90.

[24] S.Q. Gong, L.N. Niu, L.K. Kemp, C.K. Yiu, H. Ryou, Y.P. Qi, J.D. Blizzard, S. Nikonov, M.G. Brackett, R.L. Messer, C.D. Wu, J. Mao, B.L. Brister, F.A. Rueggeberg, D.D. Arola, D.H. Pashley, F.R. Tay, Quaternary ammonium silane-functionalized, methacrylate resin composition with antimicrobial activities and self-repair potential, Acta Biomater. 8 (2012) 3270-3282.

[25] S.O. Gong, D.J. Epasinghe, B. Zhou, L.N. Niu, K.A. Kimmerling, F.A. Rueggeberg, C.K. Yiu, J. Mao, D.H. Pashley, F.R. Tay, Effect of water-aging on the antimicrobial activities of an ORMOSIL-containing orthodontic acrylic resin, Acta Biomater. 9 (2013) 6964-6973.

[26] S.Q. Gong, J. Epasinghe, F.A. Rueggeberg, L.N. Niu, D. Mettenberg, C.K. Yiu, J.D. Blizzard, C.D. Wu, J. Mao, C.L. Drisko, D.H. Pashley, F.R. Tay, An ORMOSILcontaining orthodontic acrylic resin with concomitant improvements in antimicrobial and fracture toughness properties, PLoS One 7 (2012) e42355.

[27] A. Tezvergil-Mutluay, K.A. Agee, T. Uchiyama, S. Imazato, M.M. Mutluay, M. Cadenaro, L. Breschi, Y. Nishitani, F.R. Tay, D.H. Pashey, The inhibitory effects of quaternary ammonium methacrylates on soluble and matrix-bound MMPs, J. Dent. Res. 90 (2011) 535-540.

[28] F. Li, H. Majd, M.D. Weir, D.D. Arola, H.H. Xu, Inhibition of matrix metalloproteinase activity in human dentin via novel antibacterial monomer, Dent. Mater. 31 (2015) 284-292.

[29] N. Liu, F. Li, Y.J. Chen, L. Zhang, S. Lu, J.J. Kang, J.H. Chen, The inhibitory effect of a polymerisable cationic monomer on functional matrix metalloproteinases, J. Dent. 41 (2013) 1101-1108.

[30] A. Tezvergil-Mutluay, K.A. Agee, A. Mazzoni, R.M. Carvalho, M. Carrilho, I.L. Tersariol, F.D. Nascimento, S. Imazato, L. Tjäderhane, L. Breschi, F.R. Tay, D.H. Pashley, Can quaternary ammonium methacrylates inhibit matrix MMPs and cathepsins?, Dent Mater. 31 (2015) e25-32.

[31] D. Daood, C.K.Y. Yiu, M.F. Burrow, L.N. Niu, F.R. Tay, Effect of a novel quaternary ammonium silane cavity disinfectant on durability of resin-dentine bond, J. Dent. 60 (2017) 77-86.

[32] A.A. Torkelson, A.K. da Silva, D.C. Love, J.Y. Kim, J.P. Alper, B. Coox, J. Dahm, P. Kozodoy, R. Maboudian, K.L. Nelson, Investigation of quaternary ammonium silane-coated sand filter for the removal of bacteria and viruses from drinking water, J. Appl. Microbiol. 113 (2012) 1196-1207.

[33] Y.W. Cavalcanti, D.J. Morse, W.J. da Silva, A.A. Del-Bel-Cury, X. Wei, M. Wilson, P. Milward, M. Lewis, D. Bradshaw, D.W. Williams, Virulence and pathogenicity of Candida albicans is enhanced in biofilms containing oral bacteria, Biofouling. 31 (2015) 27-38.

[34] L.E. Chavez de Paz, Image analysis software based on color segmentation for characterization of viability and physiological activity of biofilms, Appl. Environ. Microbiol. 75 (2009) 1734-1739.

[35] B.M. Peters, R.M. Ward, H.S. Rane, S.A. Lee, M.C. Noverr, Efficacy of ethanol against Candida albicans and Staphylococcus aureus polymicrobial biofilms, Antimicrob. Agents Chemother. 57 (2013) 74-82.

[36] B.M. Griffiths, T.F. Watson, M. Sherriff, The influence of dentine bonding systems and their handling characteristics on the morphology and micropermeability of the dentine adhesive interface, J. Dent. 27 (1999) 63-71.

[37] P.H. D’Alpino, J.C. Pereira, N.R. Svizero, F.A. Rueggeberg, D.H. Pashley, Use of fluorescent compounds in assessing bonded resin-based restorations: a literature review, J. Dent. 34 (2006) 623-634.
[38] M. Addy, R. Handley, The effects of the incorporation of chlorhexidine acetate on some physical properties of polymerized and plasticized acrylics, J. Oral Rehabil. 8 (1981) 155-163.

[39] S. Imazato, Y. Kinomoto, H. Tarumi, S. Ebisu, F.R. Tay, Antibacterial activity and bonding characteristics of an adhesive resin containing antibacterial monomer MDPB, Dent. Mater. 19 (2003) 313-319.

[40] T. Ikeda, J. De Munck, K. Shirai, K. Hikita, S. Inoue, H. Sano, P. Lambrechts, B. Van Meerbeek, Effect of fracture strength of primer-adhesive mixture on bonding effectiveness, Dent. Mater. 21 (2005) 413-420.

[41] A.M. Sanares, A. Itthagarun, N.M. King, F.R. Tay, D.H. Pashley, Adverse surface interactions between one-bottle light-cured adhesives and chemical-cured composites, Dent. Mater. 17 (2001) 542-556.

[42] F. Abedin, B. Roughton, Q. Ye, P. Spencer, K. Camarda, Computer-aided molecular design of water compatible visible light photosensitizers for dental adhesive, Chem. Eng. Sci. 159 (2017) 131-139.

[43] Y. Delaviz, Y. Finer, J.P. Santerre, Biodegradation of resin composites and adhesives by oral bacteria and saliva: a rationale for new material designs that consider the clinical environment and treatment challenges, Dent. Mater. 30 (2014) 16-32.

[44] L. Song, R. Baney, Antibacterial evaluation of cotton textile treated by trialkoxysilane compounds with antimicrobial moiety, Text. Res. J. 5 (2010) 504-511.

[45] M. Balouiri, M. Sadiki, S.K. Ibnsouda, Methods for in vitro evaluating antimicrobial activity: A review, J. Pharm. Anal. 6 (2016) 71-79.

[46] S.S. Mo, W. Bao, G.Y. Lai, J. Wang, M.Y. Li, The microfloral analysis of secondary caries biofilm around Class I and Class II composite and amalgam fillings, BMC infect. Dis. 10 (2010) 241.

[47] R. Kaur, S. Liu, Antibacterial surface design - Contact kill, Prog. Surf. Sci. 91 (2016) 135-153.

[48] S.Y. Liu, L. Tonggu, L.N. Niu, S.Q. Gong, B. Fan, L. Wang, J.H. Zhao, C. Huang, D.H Pashley, F.R. Tay, Antimicrobial activity of a quaternary ammonium methacryloxy silicate-containing acrylic resin: a randomised clinical trial Sci. Rep. 6 (2016) 21882.

[49] D. Dufour, C.M. Lévesque, Cell death of Streptococcus mutans induced by a quorum-sensing peptide occurs via a conserved streptococcal autolysin, J. Bacteriol. 195 (2013) 105-114.

[50] N. Allocati, M. Masulli, C. Di Ilio, V. De Laurenzi, Die for the community: an overview of programmed cell death in bacteria, Cell Death Dis. 6 (2015) e1609.

[51] E. Shanker, M.J. Federle, Quorum sensing regulation of competence and bacteriocins in Streptococcus pneumoniae and mutans, Genes (Basel). 8 (2017) 15, https://doi.org/10.3390/genes801001.

[52] P.M. Scaffa, L. Breschi, A. Mazzoni, C.M. Vidal, R. Curci, F. Apolonio, P. Gobbi, D. Pashley, L. Tjäderhane, I.L. Tersariol, F.D. Nascimento, M.R. Carrilho, Codistribution of cysteine cathepsins and matrix metalloproteases in human dentin, Arch. Oral Biol. 74 (2017) 101-107.

[53] A. Mazzoni, L. Tjäderhane, V. Checchi, R. Di Lenarda, T. Salo, F.R. Tay, D.H Pashley, L. Breschi, Role of dentin MMPs in caries progression and bond stability, J. Dent. Res. 94 (2015) 241-251.

[54] O. Zitka, J. Kukacka, S. Krizkova, D. Huska, V. Adam, M. Masarik, R. Prusa, R Kizek, Matrix metalloproteinases, Curr. Med. Chem. 17 (2010) 3751-3768.

[55] Y. Kuboki, G.L. Mechanic, Comparative molecular distribution of cross-link in bone and dentin collagen. Structure-function relationships, Calcif. Tissue Int 34 (1982) 306-308.

[56] G. Turco, M. Cadenaro, T. Maravić, A. Frassetto, E. Marsich, A Mazzoni, R. Di Lenarda, F.R. Tay, D.H. Pashley, L. Breschi, Release of ICTP and CTX telopeptides from demineralized dentin matrices: Effect of time, mass and surface area Dent. Mater. 34 (2018) 452-459.

[57] W.M. Frederiks, O.R. Mook, Metabolic mapping of proteinase activity with emphasis on in situ zymography of gelatinases: review and protocols, J Histochem. Cytochem. 52 (2004) 711-722.

[58] A. Mazzoni, F.D Nascimento, M. Carrilho, I Tersariol, V. Papa, L. Tjäderhane, R. Di Lenarda, F.R. Tay, D.H. Pashley, L. Breschi, MMP activity in the hybrid layer detected with in situ zymography, J. Dent. Res. 91 (2012) 467-472.

[59] D. Umer, C.K. Yiu, M.F. Burrow, L.N. Niu, F.R. Tay, Effect of a novel quaternary ammonium silane on dentin protease activities, J. Dent. 58 (2017) 19-27.

[60] S. Imazato, S. Ma, J.H. Chen, H.H. Xu, Therapeutic polymers for dental adhesives: loading resins with bio-active components, Dent. Mater. 30 (2014) 97-104. 